\title{
Insights from an information thermodynamics analysis of a synthetic molecular motor
}

\author{
Shuntaro Amano, ${ }^{1}$ Massimiliano Esposito, ${ }^{2}$ Elisabeth Kreidt, ${ }^{1}$ David \\ A. Leigh*, ${ }^{1}$ Emanuele Penocchio* ${ }^{2}$ and Benjamin M. W. Roberts ${ }^{1}$ \\ ${ }^{1}$ Department of Chemistry, University of Manchester, Oxford Road, Manchester M13 9PL, United Kingdom \\ ${ }^{2}$ Complex Systems and Statistical Mechanics, Department of Physics and Materials \\ Science, University of Luxembourg, L-1511 Luxembourg City, G.D. Luxembourg
}

*email: david.leigh@manchester.ac.uk, emanuele.penocchio@uni.lu

Information is a physical quantity, the realisation of which transformed the physics of measurement and communication in the latter half of the 20th Century. However, the relationship and flow between information, energy and mechanics in chemical systems and mechanisms remains largely unexplored. Here we analyze a minimalist experimental example of an autonomous artificial chemically-driven molecular motor - a molecular information ratchet - in terms of information thermodynamics, a framework that quantitatively relates information to other thermodynamic parameters. This treatment reveals how directional motion is generated by free energy transfer from the chemical to the mechanical processes involving the motor. We find that the free energy transfer consists of two distinct contributions that can be considered as "energy flow" and "information flow". We identify the efficiency with which the chemical fuel powers the free energy transfer and show that this is a useful quantity with which to compare and evaluate mechanisms of, and guide designs for, molecular machines. The study provides a thermodynamic level of understanding of molecular motors that is general, complements previous analyses based on kinetics, and has practical implications for designing and improving synthetic molecular machines, regardless of the particular type of machine or chemical structure. In particular, the study confirms that, in line with kinetic analysis, power strokes do not affect the directionality of chemically-driven molecular machines. However, we also find that under some conditions power strokes can modulate the molecular motor current (how fast the components rotate), efficiency with respect to how free energy is dissipated, and the number of fuel molecules consumed per cycle. This may help explain the role of such conformational changes in biomolecular machine mechanisms and illustrates the interplay between energy and information in chemical systems.

\section{Introduction}

Understanding how and why a machine works in the way it does is crucial for optimizing designs and inventing new ones. For macroscopic machines such an understanding can be deduced from engineering principles and Newtonian physics. In contrast, at the molecular level there is no simple explanation for why individual components of biomachines move in a particular way. Is the movement of a specific amino acid in ATP synthase a key requisite for the mechanism, or does it occur incidentally as part of an evolutionary pathway that was overall successful? Biomolecular machines operate autonomously, apparently through Brownian ratchet mechanisms. ${ }^{1-5}$ They use energy and information to rectify the directionality of random thermal movements of their components so that work can be performed. This type of machine includes biological pumps and motors, in which the energy is generally provided in the form of a chemical potential gradient. ${ }^{6,7}$ Chemists are learning how to design synthetic analogues of such machines, ${ }^{8-13}$ with examples including a minimalist autonomous chemically-fueled molecular rotary motor ${ }^{14}$ (Fig. 1).

Kinetic models have proved useful ${ }^{15-17}$ in describing the behavior of molecular machines, but such analysis is inherently unable to give an account of free energy transfer from the fuel to the machine. The consideration of thermodynamics is essential to understand free energy transduction, how this drives directional motion and generates the capacity to perform work ${ }^{18}$, and, hence, how to optimize motor's design. Up to now attempts ${ }^{12,19-24}$ to design molecular motors have been led by chemical intuition, with little opportunity to reliably judge the effectiveness of a machine's design or performance unless and until it has been realized experimentally.

Recently, stochastic thermodynamics has emerged as a method for studying systems that operate at energies of the order of thermal fluctuations. ${ }^{25-28}$ The theory is valid even when systems are driven far from equilibrium $^{25,29,30}$ and has been used to study nonequilibrium nanoscale systems. ${ }^{31-36}$ A major achievement in this field was the connection made between stochastic thermodynamics and information theory ${ }^{37}$ which gave birth 
to "information thermodynamics". ${ }^{38,39}$ Information thermodynamics relates information to other thermodynamic quantities, such as free energy and entropy, and has proved particularly successful in resolving apparent thermodynamic paradoxes, such as Maxwell's demon. ${ }^{40}$

a

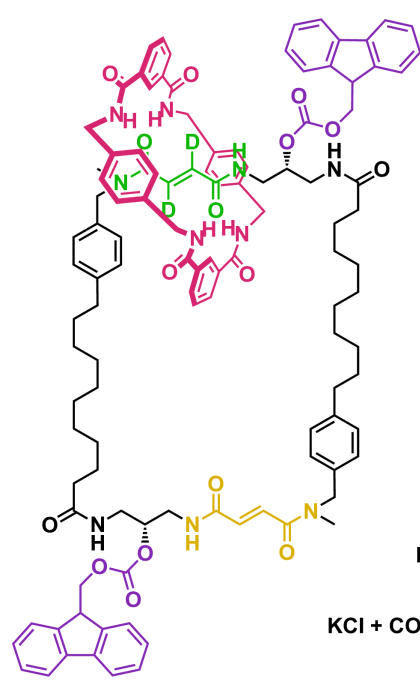

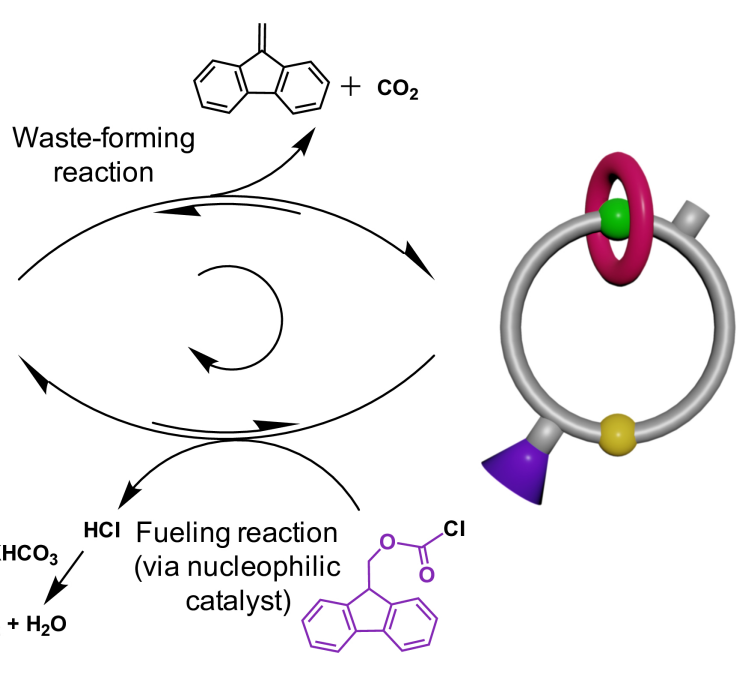

b

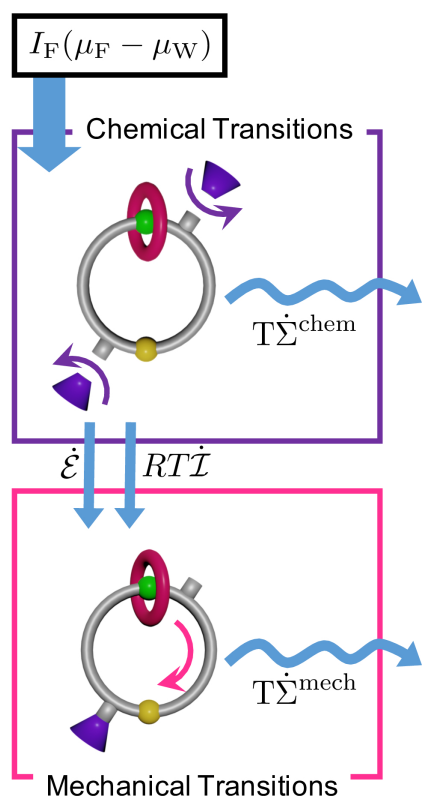

Figure 1. A Rosetta Stone for chemical (reactions and co-conformational dynamics) and information thermodynamics descriptions of a molecular motor; two distinct but complementary accounts of the processes involved in a minimalist, autonomous, chemically-fueled, molecular rotary motor. a, Chemical structure of the rotary motor and the chemical reactions involved in its operation. The motor comprises a benzylic amide macrocycle (magenta) and a track with two fumaramide binding sites (yellow, non-deuterated; green, deuterated for analytical purposes). The macrocycle randomly shuttles between the two fumaramide sites when its path is not blocked by Fmoc groups (purple). The fueling reaction consumes the fuel (Fmoc-Cl) and attaches an Fmoc group to the track, while the waste-forming reaction removes the Fmoc group (allowing passage of the macrocycle) and generates waste species (dibenzofulvene and $\mathrm{CO}_{2}$ ). The fueling reaction is catalyzed by a pyridine-based nucleophilic catalyst (e.g., 4-dimethylaminopyridine or the bulky catalyst shown in Fig. 4a). Note that both reactions are considered reversible, even when the backward reactions (i.e. regeneration of fuel via barrier removal and waste products reacting to give barrier) are extremely rare events. ${ }^{41} \mathbf{b}$, Information thermodynamics description of free energy transduction in the rotary motor. In the chemical transitions, free energy is supplied to the motor $\left(I_{\mathrm{F}}\left(\mu_{\mathrm{F}}-\mu_{\mathrm{W}}\right)\right)$, part of which is dissipated $\left(T \dot{\Sigma}^{\text {chem }}\right)$. $I_{\mathrm{F}}$ is the rate at which the motor reacts with the fuel, $\left(\mu_{\mathrm{F}}-\mu_{\mathrm{W}}\right)$ the chemical potential difference between fuel and waste species, $T$ the temperature, $\dot{\Sigma}^{\text {chem }}$ the entropy production rate of the chemical transitions. The rest of the free energy is supplied to the mechanical transitions either as energy $(\dot{\mathcal{E}})$ or information $(R T \dot{\mathcal{I}})$ flow, where $R$ is the gas constant. Mechanical transitions dissipate this free energy $\left(T \dot{\Sigma}^{\text {mech }}\right)$, generating directional motion of the macrocycle. When $\dot{\mathcal{E}}+R T \dot{\mathcal{I}}=0$, mechanical transitions are at equilibrium and no net mechanical displacement of the macrocycle can arise.

Here, we develop a quantitative understanding of the processes that drive an autonomous chemically-fueled molecular motor ${ }^{14}$ (Fig. 1) using an analysis that incorporates concepts from information thermodynamics within the framework of nonequilibrium thermodynamics of open chemical reaction networks. ${ }^{42,43}$ Contrary to common models in stochastic thermodynamics ${ }^{25,30}$, our framework neglects fluctuations and describes macroscopic ensembles of chemical species characterized by experimentally measurable concentrations. The approach is consistent with kinetic models ${ }^{16,17,44}$ but goes further by introducing a quantitative thermodynamic understanding of how autonomous molecular motors work. Two features, "information flow" and "energy flow", contribute to the transfer of free energy from the fuel to the machine that is the origin of current (i.e. the net rate of displacement of the macrocycle directionally along the track, see Box I) in the motor. The effect of changing chemical gating, ${ }^{15}$ power strokes ${ }^{45-47}$ ("a viscoelastic, free energy releasing, large-amplitude conformational change" 45,47 ), and overall rates on current and efficiency (Box I) are examined through simulations, revealing design principles for molecular motors. Particular insight is gained in terms of the role of power strokes in tuning a motor's performance while remaining consistent with core aspects of kinetic models, ${ }^{16,17,44-48}$ informing the current debate concerning the role of power strokes in biomolecular machines. ${ }^{44,46,47}$

As a result, the mechanism of operation of the rotary motor can be understood in several different ways: through chemical design, ${ }^{14}$ reaction kinetics, ${ }^{15,49}$ molecular dynamics,${ }^{50}$ and now, nonequilibrium information 
Box I. Definitions as applied to the minimalist rotary motor

$\begin{array}{ll}\text { Fueling reaction } & \text { The reaction that consumes the fuel }(\mathrm{Fmoc}-\mathrm{Cl}) \text { and attaches an Fmoc group to the motor's } \\ \text { track, generating } \mathrm{HCl} \text { as waste product. Forward }\left(k_{+\mathrm{F}}^{\mathrm{p} / \mathrm{d}}\right) \text { and backward }\left(k_{-\mathrm{F}}^{\mathrm{p} / \mathrm{d}}\right) \text { rate constants } \\ \text { may depend on the macrocycle being proximal }(\mathrm{p}) \text { or distal }(\mathrm{d}) \text { to the reaction site. }\end{array}$

thermodynamics. Accordingly, this minimalist molecular motor can act as a "Rosetta Stone" for relating these disparate frameworks ${ }^{16,17,35,44,49-51}$, aiding the translation of concepts and relationships between energy, information, kinetics and molecular structure.

\section{A bipartite chemical reaction network for the minimalist rotary motor}

The rotary motor in Fig. 1 comprises a cyclic track with two degenerate binding sites (distinguishable by one being labelled with deuterium) for a macrocycle. ${ }^{14}$ Fluorenylmethoxycarbonyl (Fmoc) groups, that sterically prevent passage of the macrocycle, can be attached to hydroxy residues on the track (Fig. 2). When only one barrier is in place, macrocycle shuttling enables exchange between two co-conformers ("co-conformers" are structures that differ in the relative positions of the components ${ }^{52}$ ) that have the macrocycle either proximal or distal to the free hydroxy group. The fueling reaction kinetically discriminates between the two co-conformers, favoring the reaction of the distal co-conformer over the proximal co-conformer. Under basic conditions, the waste-forming reaction removes barriers without any chemical gating. The chemical gating afforded by the biased fueling reaction, and the free energy supplied by the fuel-to-waste conversion, result in directional movement of the macrocycle around the track. The rotary motor can be represented by a chemomechanical network of reactions (Fig. 2) in which mechanical and chemical transitions are coupled, as in common models for biological molecular motors ${ }^{44}$ and minimal Brownian motors with external dichotomous noise ${ }^{53}$. Since no transitions enable simultaneous change in the mechanical and chemical state, this network is said to be bipartite. ${ }^{39,54}$

\section{Information thermodynamic analysis}

As detailed in the Supplementary Information (SI), the rotary motor is modelled as the isothermal open bipartite chemical reaction network ${ }^{39,42,43}$ shown in Fig. 2. The concentrations of the six motor species $2_{\mathrm{H}}, 2_{\mathrm{D}}, 1_{\mathrm{D}}^{\mathrm{H}}, 1_{\mathrm{D}}^{\mathrm{D}}, 1_{\mathrm{H}}^{\mathrm{H}}, 1_{\mathrm{H}}^{\mathrm{D}}$ (see Fig. 2 caption) evolve according to the rate constants of each reaction following massaction kinetics. The system is open because the concentrations of fuel $(\mathrm{Fmoc}-\mathrm{Cl})$ and waste $(\mathrm{HCl}-\mathrm{liberated}$ as part of the fueling reaction, and subsequently neutralized by $\mathrm{KHCO}_{3}$ that is present to produce $\mathrm{KCl} \mathrm{CO}_{2}$ and $\mathrm{H}_{2} \mathrm{O}$ - and dibenzofulvene and $\mathrm{CO}_{2}$ produced during the waste-forming reaction) species are kept constant through addition from, or removal to, an external source. An important quantity for our analysis is the chemical potential gradient between the fuel and waste species $\left(\mu_{\mathrm{Fmoc}-\mathrm{Cl}}-\mu_{\mathrm{HCl}}-\mu_{\text {dibenzofulvene }}-\mu_{\mathrm{CO}_{2}}\right)$ which we denote $\mu_{\mathrm{F}}-\mu_{\mathrm{W}}$. For any thermodynamically consistent set of parameters (see Methods), the system will 


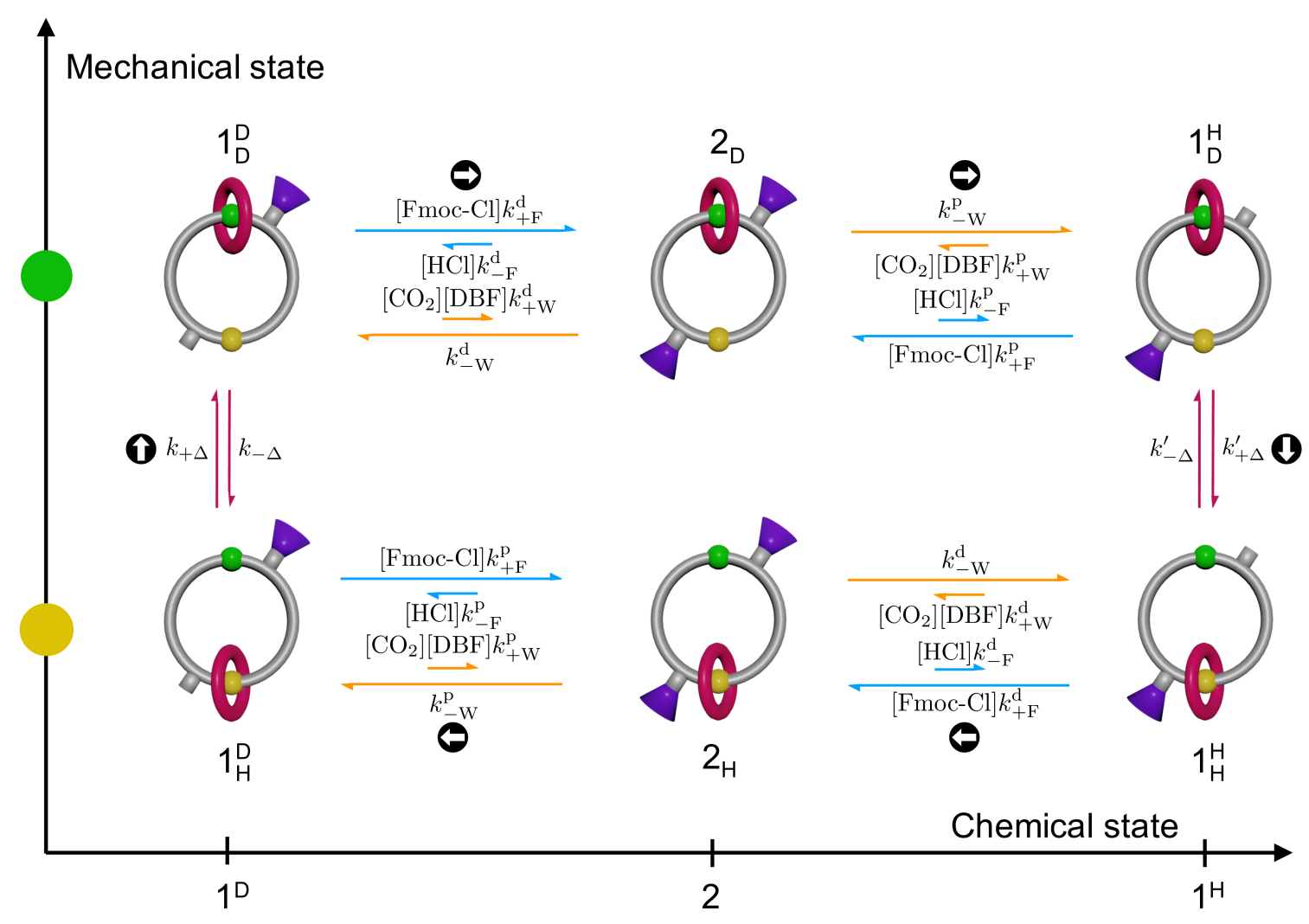

Figure 2. Rotary motor as an open and bipartite chemical reaction network. The state of the motor can be represented as a combination of two mechanical states (subscript $\mathrm{D}$ or $\mathrm{H}$, expressing whether the macrocycle is binding to the deuterated or non-deuterated site on the track) and three chemical states $\left(1^{\mathrm{D}}, 2\right.$ or $\left.1^{\mathrm{H}}\right)$. The number in the chemical state shows the number of the Fmoc groups attached to the track; the superscript H or D denotes the binding site close to the attached Fmoc group. Note that chemical states without any Fmoc group on the track are neglected. The mechanical transitions involve displacement of the macrocycle, the rate constants for which are denoted $k_{+\Delta}, k_{-\Delta}$, $k_{+\Delta}^{\prime}$ and $k_{-\Delta}^{\prime}$. Subscript $\Delta$ signifies that they are the rate constants of mechanical transitions, which are only coupled to the thermal reservoir. Rate constants with and without the prime $\left(^{\prime}\right)$ are for the interconversion between $1_{\mathrm{D}}^{\mathrm{H}}$ and $1_{\mathrm{H}}^{\mathrm{H}}$, and the interconversion between $1_{\mathrm{D}}^{\mathrm{D}}$ and $1_{\mathrm{H}}^{\mathrm{D}}$, respectively. Subscript's sign shows the direction of the transition $(+$ for the clockwise and - for the counterclockwise direction). The rate constants of the fueling reaction and its reverse are denoted as $k_{+\mathrm{F}}^{\mathrm{p} / \mathrm{d}}$ and $k_{-\mathrm{F}}^{\mathrm{p} / \mathrm{d}}$, respectively. Superscript shows whether the macrocycle is proximal (p) or distal (d) to the reacting hydroxy group. The rate constants of the waste-forming reaction and its reverse are denoted as $k_{-\mathrm{W}}^{\mathrm{p} / \mathrm{d}}$ and $k_{+\mathrm{W}}^{\mathrm{p} / \mathrm{d}}$, respectively. In the experimental rotary motor, a clockwise current across the network is generated because $k_{+\mathrm{F}}^{\mathrm{p}}<k_{+\mathrm{F}}^{\mathrm{d}}$, $k_{-\mathrm{W}}^{\mathrm{p}}=k_{-\mathrm{W}}^{\mathrm{d}}$, and free energy is supplied to the motor by the fuel-to-waste conversion. The chemical gating in the fueling reaction arises due to the steric crowding in transition states of the proximal co-conformers $\left(1_{\mathrm{D}}^{\mathrm{H}}\right.$ and $\left.1_{\mathrm{H}}^{\mathrm{D}}\right)$ compared to the distal co-conformers $\left(1_{\mathrm{D}}^{\mathrm{D}}\right.$ and $\left.1_{\mathrm{H}}^{\mathrm{H}}\right)$. Circled arrows represent the most probable (i.e., most frequent) clockwise path. DBF stands for dibenzofulvene.

evolve towards a stationary state in which the concentrations of all the motor species (i.e., all co-conformations and chemical states of the motor) are constant in time, as are the thermodynamic properties of the system. The entropy production rate (the entropy changes in the system and in the reservoirs per unit time) denoted $\dot{\Sigma}$ measures how far from equilibrium the motor operates and has to be non-negative by virtue of the Second Law of Thermodynamics. ${ }^{55}$ When multiplied by temperature $(T)$ it corresponds to the amount of free energy that is instantaneously dissipated by the motor $(T \dot{\Sigma})$. In a stationary state, the only kind of state that will be considered in this paper, all the free energy from the conversion of the fuel-to-waste is dissipated by the motor: $T \dot{\Sigma}=I_{\mathrm{F}}\left(\mu_{\mathrm{F}}-\mu_{\mathrm{W}}\right) \geq 0$, where $I_{\mathrm{F}}$ is the rate at which the fuel is consumed by the motor (we neglect the fuel-to-waste background reaction). ${ }^{43}$ This indicates that a non-null chemical potential gradient between fuel and waste species (we consider the case where $\mu_{\mathrm{F}}>\mu_{\mathrm{W}}$ ) is necessary to drive the system out of equilibrium, and produce directed motion. However, this expression gives no information as to how free energy is consumed nor the amount of dissipation specifically devoted to sustain directional motion of the components.

To obtain a more in-depth understanding, we use information thermodynamics to split the free energy dissipa- 
tion rate into two separately non-negative contributions (Fig. 1b), one due to the chemical transitions $\left(T \dot{\Sigma}^{\text {chem }}\right.$ ) and the other due to the mechanical transitions $\left(T \dot{\Sigma}^{\text {mech }}\right)$. This is possible since the Fmoc-motor chemical reaction network is bipartite: ${ }^{39}$

$$
T \dot{\Sigma}=\overbrace{I_{\mathrm{F}}\left(\mu_{\mathrm{F}}-\mu_{\mathrm{W}}\right)-\dot{\mathcal{E}}-R T \dot{\mathcal{I}}}^{T \dot{\Sigma}^{\text {chem }} \geq 0}+\overbrace{\dot{\mathcal{E}}+R T \dot{\mathcal{I}}}^{T \dot{\Sigma}^{\text {mech }} \geq 0} \geq 0 .
$$

This dissection of terms in equation (1) underlines that the free energy supplied by the fuel is only partially dissipated via the chemical processes as $T \dot{\Sigma}^{\text {chem }}$ while the remaining part is transferred to the mechanical processes and dissipated as $T \dot{\Sigma}^{\text {mech }}$. This transfer of free energy is composed of a standard free energy part, denoted "energy flow":

$$
\dot{\mathcal{E}}=J\left(\mu_{1_{\mathrm{H}}^{\mathrm{D}}}^{\circ}-\mu_{1_{\mathrm{D}}^{\mathrm{D}}}^{\circ}+\mu_{1_{\mathrm{D}}^{\mathrm{H}}}^{\circ}-\mu_{1_{\mathrm{H}}^{\mathrm{H}}}^{\circ}\right)
$$

and a mutual information part, 37 denoted "information flow":

$$
R T \dot{\mathcal{I}}=J R T \log \frac{\left[1_{\mathrm{H}}^{\mathrm{D}}\right]\left[1_{\mathrm{D}}^{\mathrm{H}}\right]}{\left[1_{\mathrm{D}}^{\mathrm{D}}\right]\left[1_{\mathrm{H}}^{\mathrm{H}}\right]}
$$

where $R$ is the gas constant and $J$ is the stationary clockwise (as viewed in Fig. 2) current at which the motor operates (see Box I). This current can be expressed as

$$
J=k_{+\Delta}\left[1_{\mathrm{H}}^{\mathrm{D}}\right]-k_{-\Delta}\left[1_{\mathrm{D}}^{\mathrm{D}}\right]=k_{+\Delta}^{\prime}\left[1_{\mathrm{D}}^{\mathrm{H}}\right]-k_{-\Delta}^{\prime}\left[1_{\mathrm{H}}^{\mathrm{H}}\right]=\Gamma\left(K_{\mathrm{r}}-1\right) .
$$

where both $\Gamma$ and $K_{\mathrm{r}}$ are positive quantities (for derivation, see SI section V B4), the latter denoting the ratcheting constant (recently applied in the context of dissipative self-assembly ${ }^{17,49}$ ) which quantifies the kinetic asymmetry of the motor ${ }^{15,16,56}$ (see Box I).

Under the experimental conditions in which the motor was originally operated, ${ }^{14}$ the rotary motor is driven purely by information flow as the macrocycle binds with equal affinity to fumaramide stations adjacent to a hydroxy group and an Fmoc group (within the detection limits of ${ }^{1} \mathrm{H}$ nuclear magnetic resonance (NMR) measurements), so there is no energy flow. However, when the standard chemical potentials of the distal and proximal co-conformers differ, energy flow arises according to equation (2). An example of this could arise if there was, say, a stabilizing interaction between the macrocycle and the Fmoc group, which would lead to an increase in energy of the macrocycle upon removal of the Fmoc-barrier close to it. As a consequence, the standard chemical potential would decrease, leading to the release of heat, each time net mechanical displacement occurs in the forward direction. This fits the definition of a "power stroke" ${ }^{45-47}$ (see Box I). Therefore, according to equation (2), the energy flow accounts for the part of the fueling free energy that contributes to destabilizing the macrocycle during chemical transitions and which is subsequently dissipated in a power stroke.

Mutual information quantifies the correlation between the two parts of a bipartite system ${ }^{39,54}$, here the chemical and the mechanical states. For instance, when $\left[1_{\mathrm{D}}^{\mathrm{H}}\right]$ (the concentration of the species $1_{\mathrm{D}}^{\mathrm{H}}$ ) and $\left[1_{\mathrm{H}}^{\mathrm{D}}\right]$ are larger than $\left[1_{\mathrm{H}}^{\mathrm{H}}\right]$ and $\left[1_{\mathrm{D}}^{\mathrm{D}}\right]$, respectively, a correlation is present between the mechanical and chemical states: when the motor's chemical state is $1^{\mathrm{H}}$, its mechanical state is more likely to be $\mathrm{D}$ than $\mathrm{H}$. Likewise, when the motor's chemical state is $1^{\mathrm{D}}$, its mechanical state is more likely to be $\mathrm{H}$ than $\mathrm{D}$. A concentration distribution with this kind of correlation has smaller (Shannon-like $\left.{ }^{37}\right)$ entropy than one without $\left(\left[1_{\mathrm{D}}^{\mathrm{H}}\right]\right.$ and $\left[1_{\mathrm{H}}^{\mathrm{D}}\right]$ equal to $\left[1_{\mathrm{H}}^{\mathrm{H}}\right]$ and $\left[1_{\mathrm{D}}^{\mathrm{D}}\right]$, respectively). Therefore, correlation between the mechanical and chemical states (mutual information) generates an entropic driving force for a directional current (from $\left[1_{\mathrm{D}}^{\mathrm{H}}\right]$ to $\left[1_{\mathrm{H}}^{\mathrm{H}}\right]$ and from $\left[1_{\mathrm{H}}^{\mathrm{D}}\right]$ to $\left[1_{\mathrm{D}}^{\mathrm{D}}\right]$ ). From the thermodynamic viewpoint, mutual information constitutes the entropic contribution of the free energy that comes from the fuel. As mutual information is constant in the stationary state, changes in mutual information due to the chemical processes and mechanical processes are balanced ${ }^{39}$. If, as in the above situation, $\dot{\mathcal{I}}>0$, the chemical transitions are producing mutual information that is consumed by the mechanical ones. Therefore, according to equation (3), the information flow accounts for the part of fueling free energy that contributes to increasing the system's mutual information during chemical transitions and which is subsequently erased by mechanical shuttling.

Regimes where the free energy supplied by the chemical to the mechanical processes is exclusively due to the information flow $(R T \dot{\mathcal{I}})$, and thus lack any energy flow $(\dot{\mathcal{E}})$, are denoted as pure Maxwell demon regimes. ${ }^{39}$

This analysis demonstrates that the free energy supplied to the mechanical processes by the energy and information flows, $R T \dot{\mathcal{I}}+\dot{\mathcal{E}}$, is the origin of net directional motion of the macrocycle around the track. Indeed, in the absence of such flow $(R T \dot{\mathcal{I}}+\dot{\mathcal{E}}=0)$ the mechanical transitions are at thermodynamic equilibrium $\left(\dot{\Sigma}^{\text {mech }}=0\right)$, meaning a zero directional shuttling current $(J=0$; see equation $(4))$. From an information thermodynamics perspective, the molecular motor operates by using chemical processes to transduce the free 
energy supplied by the fuel into the free energy supplied to the mechanical processes. The efficiency of this transduction is the ratio of the latter to the former and is bounded between zero and one due to the nonnegativity of $\dot{\Sigma}^{\text {chem: }}$

$$
0 \leq \eta=\frac{R T \dot{\mathcal{I}}+\dot{\mathcal{E}}}{I_{\mathrm{F}}\left(\mu_{\mathrm{F}}-\mu_{\mathrm{W}}\right)}=1-\frac{T \dot{\Sigma}^{\text {chem }}}{I_{\mathrm{F}}\left(\mu_{\mathrm{F}}-\mu_{\mathrm{W}}\right)} \leq 1
$$

In this setup, the energy and information flows are entirely dissipated by the shuttling of the macrocycle as $T \dot{\Sigma}^{\text {mech }}$. If the mechanical steps of the motor were to work against a force (for example if a load were attached to the macrocycle), $T \dot{\Sigma}^{\text {mech }}$ would incorporate a negative work term in addition to $R T \dot{\mathcal{I}}+\dot{\mathcal{E}}$, which could serve to define the efficiency of the energy and information flows being converted into output work, instead of just being dissipated. The energy and information flows would thus constitute the maximum work output that can be delivered by the motor. A traditional thermodynamic analysis of such a motor ${ }^{25,26,57}$ would exclusively focus on the efficiency with which the input free energy supplied by the fuel-to-waste chemical potential gradient is converted into output work, thus over-estimating the maximum work output as the overall free energy input $I_{F}\left(\mu_{\mathrm{F}}-\mu_{\mathrm{W}}\right)$. The present approach refines this analysis by showing how the input-to-output transduction is mediated by the free energy transfer within the motor, whose efficiency $\eta$ (equation (5)) limits the maximum work output potentially deliverable by the motor. It also formally defines a thermodynamic efficiency that can be applied to motors while they perform no appreciable output work, as is the case for most of the synthetic molecular motors made to date, and can serve to compare the efficiencies of their operation.

The framework we have outlined can also be used to re-derive previous results obtained using kinetic arguments as a consequence of the Second Law of Thermodynamics in bipartite systems. In the SI (section V B), we show that the condition $T \dot{\Sigma}^{\text {mech }}=0$ implies $K_{\mathrm{r}}=1$, while the condition $T \dot{\Sigma}^{\text {mech }}>0$ implies $K_{\mathrm{r}} \neq 1$, with forward movement when $K_{\mathrm{r}}>1$. This shows how the nonequilibrium thermodynamic framework, which focuses on energetic aspects quantified by the dissipation $T \dot{\Sigma}^{\text {mech }}$, is consistent with previous analysis ${ }^{15-17,49}$ focusing on kinetic aspects quantified by $K_{\mathrm{r}}$, which determines the sign of the current $J$ according to equation (4). This reiterates the effectiveness of this information-thermodynamics-based approach and, again, demonstrates the usefulness of this minimalist molecular motor as a Rosetta Stone for the translation of meaning and understanding between different frameworks for describing phenomena.

Previously, ${ }^{17}$ the ratcheting constant has also been related to the ability of a dissipative self-assembly system to store free energy, but this connection is only valid in an operating regime where chemical transitions are significantly faster than mechanical ones. The information thermodynamics framework offers a general understanding of dissipative chemical systems and establishes limits to the maximum work deliverable by them that are valid in any operating regime, thanks to concepts such as the efficiency $\eta$ introduced in equation (5).

\section{Design principles for molecular motors}

To demonstrate the use of our framework as a design tool, we explored the effects of altering the design features of the rotary motor on its current and efficiency (see SI section VI). Basing the simulations on experimentally derived parameters $^{14}$ (see SI section VI A), we established that under the experimental conditions employed, the rotary motor is driven purely by information flow as only the macrocycle distribution, rather than binding site affinity (within the detection limits of ${ }^{1} \mathrm{H}$ nuclear magnetic resonance (NMR) measurements), is altered during operation. Under experimental conditions ([motor $]=10 \mathrm{mM},[\mathrm{Fmoc}-\mathrm{Cl}]=30 \mathrm{mM}$, which is sustained by constant addition, [bulky catalyst] $=50 \mathrm{mM},\left[\mathrm{Et}_{3} \mathrm{~N}\right]=15 \mathrm{mM},\left[\mathrm{KHCO}_{3}\right]=200 \mathrm{mM} \mathrm{CH}_{2} \mathrm{Cl}_{2}$, room temperature), ${ }^{14}$ the current was estimated to be $2.1 \cdot 10^{-8} \mathrm{~mol} \mathrm{dm}^{-3} \mathrm{~s}^{-1}$, requiring an average of seven fuel molecules per cycle per motor, though only $10^{-6} \%$ of the free energy provided by the fuel is used to sustain the current (efficiency, $\eta=10^{-8}$, see equation (5)).

\section{Kinetic modifications}

Varying model parameters allows consideration of the effects of potential structural and chemical changes on the rotary motor (SI section VI B1). Greater chemical gating for either the fueling (Fig. 3a, b) or waste-forming reaction (SI section VI B2) increases the current and efficiency by increasing information flow. The former has been achieved by increasing the steric bulk of the barrier-formation catalyst ${ }^{14,58,59}$ (Fig. 4a) and the latter by catalysis of barrier removal by a proximal macrocycle (Fig. 4b) ${ }^{24}$ Gating of both fueling and waste-forming reactions (SI section VI B3) was recently demonstrated in a [2] rotaxane information ratchet. ${ }^{24}$ Inverting chemical gating is predicted to reverse the direction of the motor and could be achieved if the macrocycle activates, rather 
than hinders, proximal barrier formation (Fig. 4c). In the absence of a kinetic preference, or when gating from the fueling and waste-forming reactions cancel out, the motor stops working, as this precludes information flow and hence mechanical dissipation $\left(\dot{\Sigma}^{\text {mech }}=0\right)$. These results are consistent with kinetic models where kinetic asymmetry predicts the direction of the current. ${ }^{15-17}$
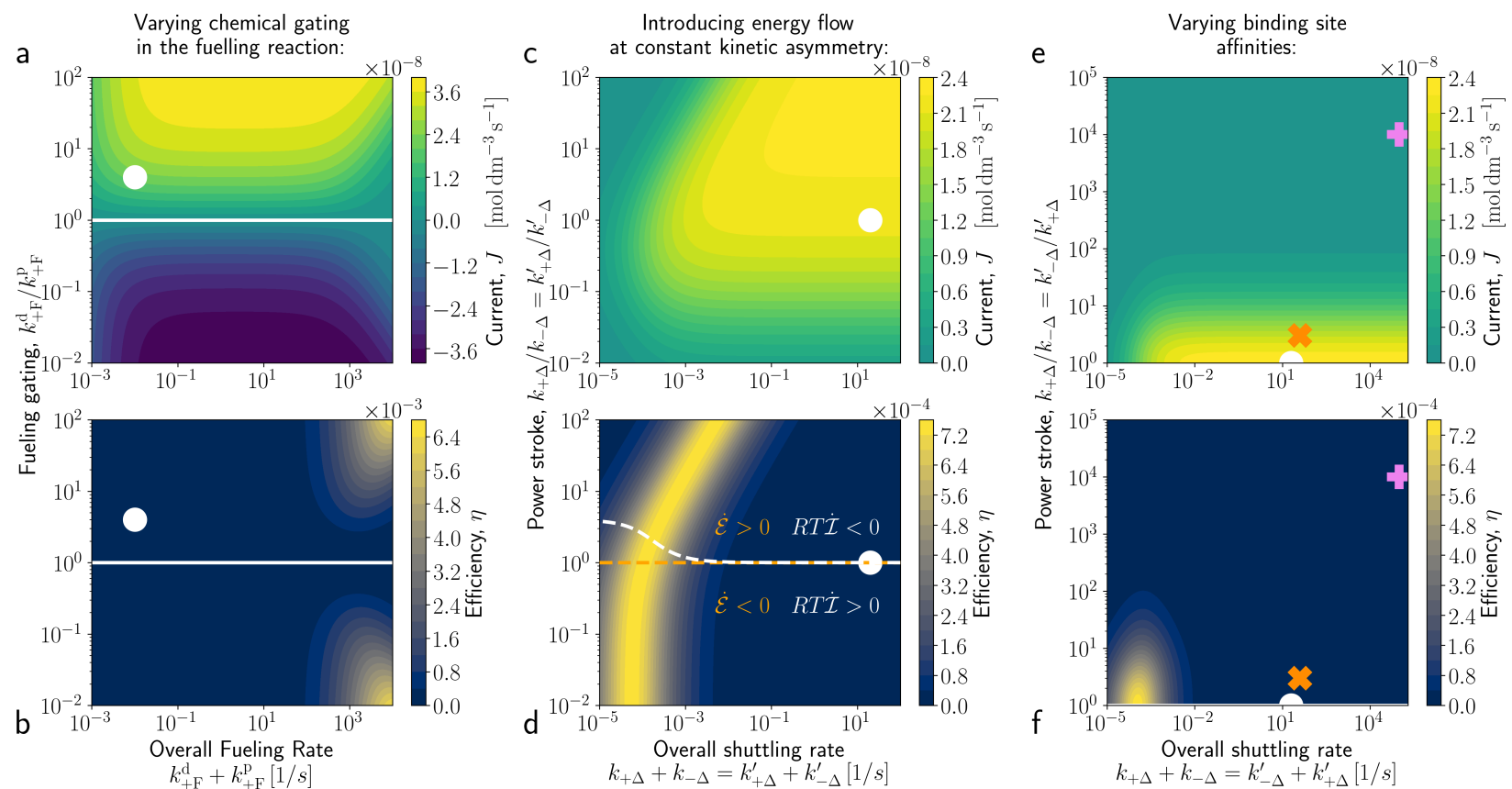

Figure 3. Numerical simulations of different molecular motor design modifications. Simulated current $(J$, top) and efficiency ( $\eta$, bottom). Solid white lines indicate the regions of null current (and efficiency). White circles indicate the approximate parameters of the experimental rotary motor, giving a current of $2.1 \cdot 10^{-8} \mathrm{~mol} \mathrm{dm}^{-3} \mathrm{~s}^{-1}$ and efficiency of $10^{-6} \%$. Directional motion in the experimental rotary motor arises because distal fueling reactions are $\sim 4 \times$ faster than proximal fueling reactions (chemical gating, $k_{+\mathrm{F}}^{\mathrm{d}} / k_{+\mathrm{F}}^{\mathrm{p}} \approx 4$ ), due to steric hindrance of the proximal transition state, while there was no chemical gating of waste-forming reactions $\left(k_{-\mathrm{W}}^{\mathrm{p}} / k_{-\mathrm{W}}^{\mathrm{d}} \approx 1\right)$. $\mathbf{a}, \mathbf{b}$, Overall rate of the fueling reactions (x-axis) and fueling gating (y-axis) are varied. $\mathbf{c}, \mathbf{d}$, Overall shuttling rate (x-axis) and the ratio of forward and backward shuttling rates (indicative of power stroke magnitude, see Methods) are varied. Power strokes are introduced in a way that induces energy flow $(\dot{\mathcal{E}})$ while keeping $K_{\mathrm{r}}$ constant. Below the dashed white lines, information flow $(R T \dot{\mathcal{I}})$ is positive and thus contributes to mechanical dissipation $\dot{\Sigma}^{\text {mech }}$, while above these lines, information flow is negative, requiring the motor to be driven by positive energy flow $(\dot{\mathcal{E}}>0)$. Negative energy flow is found below the orange dashed line. e,f, Overall shuttling rate ( $\mathrm{x}$-axis) and ratio of shuttling rate between stations (y-axis) are varied. When this ratio diverges from one (indicating non-degenerate binding sites, see Methods), both the current and efficiency drop. The pink + symbol indicates the position expected by changing one fumaramide group to a succinamide. ${ }^{60}$ The orange $\times$ symbol indicates a position with a 3:1 difference in binding site affinity for the macrocycle.

Power stroke modifications

The relevance of power strokes in molecular machinery is contentious as power strokes are often experimentally observed in biological molecular motors, ${ }^{44-46}$ but according to analysis based on kinetic asymmetry, the magnitude of the free energy released by such conformational changes does not affect the properties of chemically-driven molecular machines such as directionality and stopping force, and cannot improve the efficiency of a motor to work against an external force. ${ }^{47}$ To reconcile differing viewpoints as to the significance of power strokes in molecular machines, we attempted to use our framework to understand the ways in which a power stroke can affect a molecular motor while staying kinetically and thermodynamically consistent. Power strokes can be used to induce energy flow (equation (2)) and, in principle, could be added to the rotary motor by introducing attractive interactions between the barrier and the macrocycle (Fig. 4d), stabilizing distal co-conformers $1_{\mathrm{D}}^{\mathrm{D}}$ and $1_{\mathrm{H}}^{\mathrm{H}}$, or by adding repulsive interactions between the free barrier site and the macrocycle (Fig. 4e), destabilizing proximal co-conformers $1_{\mathrm{H}}^{\mathrm{D}}$ and $1_{\mathrm{D}}^{\mathrm{H}}$. Our simulations show that power strokes can change the magnitude of the current and efficiency of internal energy transduction (as defined in equations (4) and (5), 
respectively) despite kinetic asymmetry remaining unaltered (see Methods, SI section VI B4). This is because, while not altering $K_{\mathrm{r}}$, power strokes can still increase the value of $\Gamma$ in equation (4), reflecting their ability to favor forward cycles by inducing energy flow out of equlibrium (Fig. 3c, d). However, power strokes cannot drive directional motion in the absence of kinetic asymmetry $\left(R T \dot{\mathcal{I}}=-\dot{\mathcal{E}}\right.$ when $\left.K_{\mathrm{r}}=1\right)$, nor can they invert directionality while the kinetic asymmetry remains constant. In these simulations, altering power strokes and kinetic asymmetry together (SI section VI B5) gave the greatest simulated efficiency suggesting that modifying both aspects may be important for optimizing the design of synthetic molecular motors. We note that improvements that occur through power strokes arise from the induced energy flow rather than from any special role for the energetically downhill nature of the power stroke in determining the motor's behaviour. As our analysis shows, a prerequisite for the motor's operation is the availability of free energy to dissipate through mechanical motion. Therefore, any design feature that enhances free energy transduction from the chemical to the mechanical transitions could equally well foster improvements in performance.

If power strokes cancel out over the motor cycle, then no net energy flow is introduced: free energy gained from one mechanical transition is lost in the other (see Methods, SI section VI B6). This could be realized in molecular form by using non-degenerate binding sites, for example, changing one fumaramide site to a more weakly-binding succinamide unit (Fig. 3e, f and 4f) with a typical difference in binding energy of $23 \mathrm{~kJ} \mathrm{~mol}^{-1}$ (pink + symbol, Fig. 3e, f, equilibrium distribution $>99: 1)^{60}$ under experimental conditions similar to those used for rotary motor operation. Kinetic asymmetry remains unaltered in this scenario and, correspondingly, the direction of the motor could not be inverted in the simulation. ${ }^{15-17}$ However, our analysis suggests that such a change would be sufficient to effectively stall the motor, if operated under the original experimental conditions, despite the unchanged kinetic asymmetry. The simulations predict that, with power strokes cancelling out, any change from degenerate binding sites lowers the current and efficiency, though a smaller difference would leave the motor functional, albeit less effective. A 3:1 bias ${ }^{58}$ (orange $\times$ symbol, Fig. 3e, f,) is predicted to reduce the current by $\sim 20 \%$, rendering a design with non-degenerate binding sites plausible but less effective than a motor with binding sites of equal affinities.

In all of the cases considered, the highest efficiencies are predicted for when the rates of all the forward processes are approximately equal, leading to a cycle with no single rate-limiting step. Rate-limiting mechanical steps promote futile cycles, in which fuel is consumed without taking a forward step, as the unfavorable fueling reaction is kinetically favored over shuttling, decreasing both current and efficiency. Rate-limiting chemical reactions decrease the information flow in the steady-state since fast shuttling hinders the generation of a concentration bias compared to mechanical equilibrium, resulting in lower thermodynamic efficiency without reducing current or substantially changing fuel consumption per cycle. The strong dependence of efficiency on shuttling rate indicates that, like macroscopic engines, the efficiency of a motor will be dependent on the load it is working against. ${ }^{35}$ To use molecular machines most efficiently, they must either be tailored to the job they perform — such as using diesel engines for heavy loads — or they must use the equivalent of gears for macroscopic engines, to ensure they are working under optimal conditions.

\section{Conclusions}

Information thermodynamics-based analysis of a minimalist autonomous chemically-driven molecular motor shows how information and energy flow, the two components of free energy transfer from chemical to mechanical transitions, enable the generation of directional motion from free energy supplied by a chemical fuel. The experimental rotary motor is a pure "chemical Maxwell's demon", as information flow is the sole driving force. However, energy flow could potentially be introduced using power strokes, one of several design variations explored using our model. The predicted effect of energy flow is in line with observations made in biological motors $^{44}$ and contributes to the ongoing debate regarding the role of power strokes in molecular motors ${ }^{46-48}$. Information thermodynamics confirms that, in line with kinetic analysis, power strokes do not affect some key properties of chemically-driven molecular machines such as directionality. However, the magnitude of power strokes is able to affect the magnitude of the current (how fast the motor components rotate), the efficiency in terms of how free energy is dissipated, and the number of fuel molecules consumed per cycle. But these results should not be misinterpreted as supporting special significance of power strokes compared to other processes in the chemomechanical cycle. The information thermodynamics framework used in this paper should be generally applicable to other types of synthetic molecular machines, such as non-autonomous ${ }^{10,12}$ and light-driven ${ }^{8,9,11}$ motors, providing a quantitative basis through which to compare molecular machine designs. Additionally, it could, in principle, be extended to other types of (supra)molecular systems (such as dissipative self-assembly ${ }^{17}$ ) powered by chemical engines ${ }^{61}$. We have uncovered for the first time significant roles for 'energy flow' and 'information flow' in the mechanism of the transduction of free energy from chemical reactions by molecular machinery, although the exact nature of the connection of energy and information flow to energy and information 
a

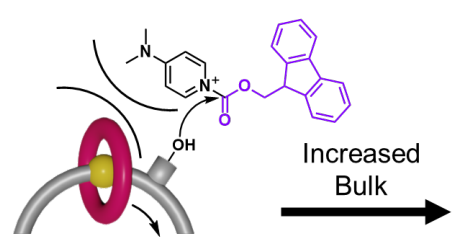

b

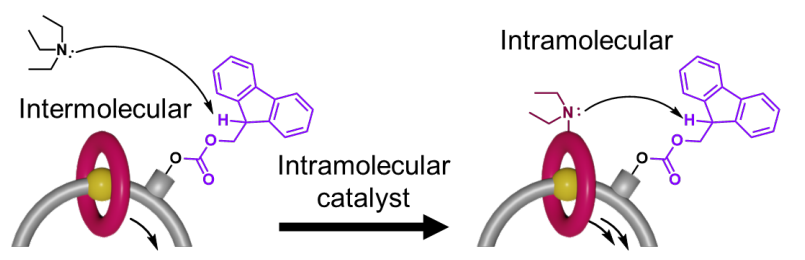

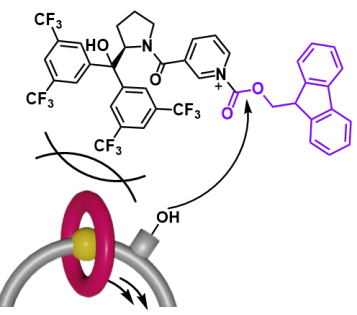

西

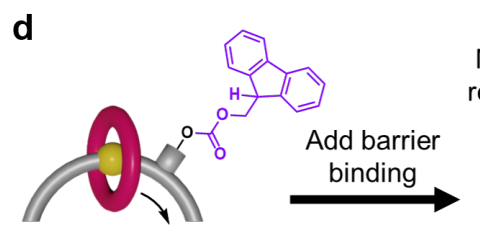

e

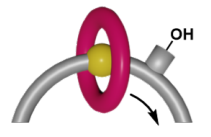

f

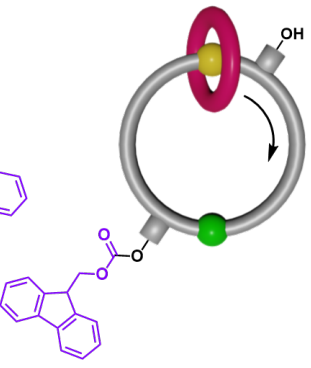

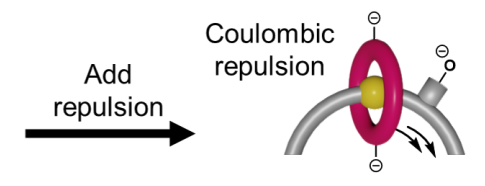

Fumaramide

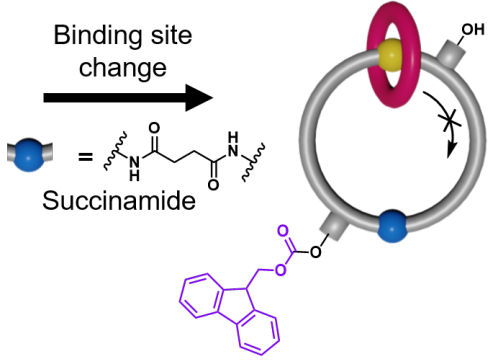

Figure 4. Potential ways of achieving different molecular motor design modifications. a, Increasing the steric bulk of the catalyst could be used to increase the fueling chemical gating by slowing the undesired proximal reaction. $\mathbf{b}$, Addition of a catalyst for the waste-forming reaction to the macrocycle could increase waste-formation chemical gating by increasing the rate of the proximal waste-forming reaction. c, Addition of a catalyst to the macrocycle that accelerates rather than hinders proximal barrier formation could negate chemical gating arising from steric hindrance, leading to an inversion of the directionality of the motor. $\mathbf{d}$, Allowing the formation of a complex that binds the barrier and the macrocycle could allow the introduction of a power stroke by stabilizing the co-conformer in which the macrocycle is adjacent to the barrier. e, Conversely, a power stroke might be introduced by destabilizing the co-conformer with the macrocycle adjacent to the site with no barrier, for example by introduction of coulombic repulsion. f, Swapping one fumaramide binding site for a succinamide should, in principle, be sufficient to stall the rotary motor under the previous experimental operating conditions.

ratcheting ${ }^{1,62}$ remains to be clarified. The minimalist autonomous chemically-driven molecular motor acts as a Rosetta Stone for relating energy, information, kinetics and molecular structure by aiding the translation of concepts and relationships between the 'languages' (i.e. frameworks) of chemical kinetics, thermodynamics and chemical reactions.

\section{Methods}

Wegscheider's conditions: To ensure that the system reaches thermodynamic equilibrium (detailed balance), when there is no chemical potential gradient between fuel and waste species $\left(\mu_{\mathrm{F}}-\mu_{\mathrm{W}}=0\right)$, the rate constants must satisfy Wegscheider's conditions. ${ }^{42}$ These are equivalent to the constraints on the rate constants imposed in previous kinetic analyses ${ }^{4,16,48}$ and dictate that the product of the forward rate constants along each independent cyclic pathway of reactions in the network, with neither net consumption nor net production of fuel or waste species, must equal the product of the corresponding backward rate constants:

$$
\begin{aligned}
& k_{-\mathrm{F}}^{\mathrm{p}} k_{+\Delta} k_{+\mathrm{F}}^{\mathrm{d}} k_{-\mathrm{F}}^{\mathrm{p}} k_{+\Delta}^{\prime} k_{+\mathrm{F}}^{\mathrm{d}}=k_{+\mathrm{F}}^{\mathrm{p}} k_{-\Delta} k_{-\mathrm{F}}^{\mathrm{d}} k_{+\mathrm{F}}^{\mathrm{p}} k_{-\Delta}^{\prime} k_{-\mathrm{F}}^{\mathrm{d}} \\
& k_{-\mathrm{W}}^{\mathrm{p}} k_{+\Delta} k_{+\mathrm{W}}^{\mathrm{d}} k_{-\mathrm{W}}^{\mathrm{p}} k_{+\Delta}^{\prime} k_{+\mathrm{W}}^{\mathrm{d}}=k_{+\mathrm{W}}^{\mathrm{p}} k_{-\Delta} k_{-\mathrm{W}}^{\mathrm{d}} k_{+\mathrm{W}}^{\mathrm{p}} k_{-\Delta}^{\prime} k_{-\mathrm{W}}^{\mathrm{d}},
\end{aligned}
$$

see SI for derivation. These conditions were always imposed in numerical simulations to guarantee thermodynamic consistency. ${ }^{41}$

Local Detailed Balance: In Fig. 3, variations in the power stroke magnitude have been related to changes in the ratio of shutting rate constants by virtue of the so-called "principle of local detailed balance" (see below), 
which relates the log-ratio of forward and backward rate constants of a single chemical reaction to the difference in standard chemical potentials between its reagents and products ${ }^{42}$ (see SI equation S.33). For instance, it implies the relation

$$
R T \log \left(\frac{k_{+\Delta} k_{+\Delta}^{\prime}}{k_{-\Delta} k_{-\Delta}^{\prime}}\right)=\mu_{1_{\mathrm{H}}^{\mathrm{D}}}^{\circ}-\mu_{1_{\mathrm{D}}^{\mathrm{D}}}^{\circ}+\mu_{1_{\mathrm{D}}^{\mathrm{H}}}^{\circ}-\mu_{1_{\mathrm{H}}^{\mathrm{H}}}^{\circ},
$$

which was employed in numerical simulations.

In addition, Wegscheider's conditions (equations (M1a) and (M1b)) imply that a variation in the power stroke magnitude must always be compensated by a variation in the fueling and waste-forming rate constants, since the following constraint (equation (M3)) must always hold for thermodynamic consistency:

$$
\frac{k_{+\Delta}}{k_{-\Delta}} \cdot \frac{k_{+\Delta}^{\prime}}{k_{-\Delta}^{\prime}}=\frac{k_{+\mathrm{F}}^{\mathrm{p}} k_{-\mathrm{F}}^{\mathrm{d}}}{k_{-\mathrm{F}}^{\mathrm{p}} k_{+\mathrm{F}}^{\mathrm{d}}} \cdot \frac{k_{+\mathrm{W}}^{\mathrm{p}} k_{-\mathrm{W}}^{\mathrm{d}}}{k_{-\mathrm{W}}^{\mathrm{p}} k_{+\mathrm{W}}^{\mathrm{d}}} .
$$

When energy-flow-inducing power strokes were introduced in Fig. 3c and d, the constraint in equation (M3) was imposed by changing rate constants $k_{+\mathrm{W}}^{\mathrm{p}}$ and $k_{+\mathrm{F}}^{\mathrm{p}}$ according to variations in shuttling rate constants. By doing so, kinetic asymmetry $\left(K_{\mathrm{r}}\right)$ is not altered during the simulation, but the magnitude of the current in equation (4) can still change by virtue of alterations in the value of the positive factor $\Gamma$ (for its mathematical expression, see SI section V B4). Instead, in Supplementary Fig. S.5 rate constants $k_{+\mathrm{W}}^{\mathrm{p}}$ and $k_{-\mathrm{F}}^{\mathrm{p}}$ were changed to vary energy flow and $K_{\mathrm{r}}$ together. Experimentally, this could correspond to introducing an interaction between the macrocycle and the Fmoc group that affects (Supplementary Fig. S.5) or not (Fig. 3c and d) the transition state of the proximal fueling reaction, without affecting the transition state of the proximal waste-forming reaction. Note that, when binding affinities are modified as in Fig. 3e and f, the left-hand side of equation (M3) stays constant and the constraint is automatically satisfied.

We end by noting that the terminology "local detailed balance" comes from statistical physics ${ }^{63}$, where it has become the central concept to formulate thermodynamically consistent dynamics ${ }^{64,65}$. Its chemical counterpart $^{42}$ (see SI equation S.33) is fully equivalent to usual conditions imposed on the rate constants to ensure that microscopic reversibility holds ${ }^{47}$ (see for instance equation 5 in reference 16). We note that use of the term "local detailed balance" in this context is considered contentious by some. ${ }^{16}$ For a more detailed discussion see SI section III.

\section{Acknowledgements}

We thank the European Research Council (ERC Consolidator Grant n. 681456 to M.E. and funding E.P.; ERC Advanced Grant n. 786630 to D.A.L), the FQXi foundation, project "Information as a fuel in colloids and superconducting quantum circuits" (grant FQXi-IAF19-05 to M.E.), the Engineering and Physical Sciences Research Council (EPSRC; grant EP/P027067/1 to D.A.L.), the Deutsche Forschungsgemeinschaft (DFG) for a postdoctoral fellowship to E.K., and the University of Manchester and EPSRC for PhD studentships to S.A. and B.M.W.R.. D.A.L. is a Royal Society Research Professor. We thank R. D. Astumian for valuable discussions regarding the science in this study as well as robust debate regarding the use of the term 'local detailed balance' within the stochastic thermodynamics community.

\section{REFERENCES}

${ }^{1}$ Chatterjee, M. N., Kay, E. R. \& Leigh, D. A. Beyond switches: Ratcheting a particle energetically uphill with a compartmentalized molecular machine. J. Am. Chem. Soc. 128, 4058-4073 (2006).

2 Coskun, A., Banaszak, M., Astumian, R. D., Stoddart, J. F. \& Grzybowski, B. A. Great expectations: can artificial molecular machines deliver on their promise? Chem. Soc. Rev. 41, 19-30 (2012).

3 Erbas-Cakmak, S., Leigh, D. A., McTernan, C. T. \& Nussbaumer, A. L. Artificial molecular machines. Chem. Rev. 115, 10081-10206 (2015).

${ }^{4}$ Qiu, Y., Feng, Y., Guo, Q.-H., Astumian, R. D. \& Stoddart, J. F. Pumps through the ages. Chem 6, 1952 - 1977 (2020).

5 Aprahamian, I. The future of molecular machines. ACS Cent. Sci. 6, 347-358 (2020).

${ }^{6}$ Howard, J. Mechanics of Motor Proteins and the Cytoskeleton (Oxford University Press, Oxford, 2001).

7 Astumian, R. D., Mukherjee, S. \& Warshel, A. The physics and physical chemistry of molecular machines. ChemPhysChem 17, 1719-1741 (2016).

${ }^{8}$ Koumura, N., Zijlstra, R. W. J., van Delden, R. A., Harada, N. \& Feringa, B. L. Light-driven monodirectional molecular rotor. Nature 401, 152-155 (1999). 
${ }^{9}$ Serreli, V., Lee, C.-F., Kay, E. R. \& Leigh, D. A. A molecular information ratchet. Nature 445, 523-527 (2007).

10 Cheng, C. et al. An artificial molecular pump. Nat. Nanotechnol. 10, 547-553 (2015).

11 Ragazzon, G., Baroncini, M., Silvi, S., Venturi, M. \& Credi, A. Light-powered autonomous and directional molecular motion of a dissipative self-assembling system. Nat. Nanotechnol. 10, 70-75 (2015).

12 Erbas-Cakmak, S. et al. Rotary and linear molecular motors driven by pulses of a chemical fuel. Science 358, 340-343 (2017).

13 Amano, S., Fielden, S. D. P. \& Leigh, D. A. A catalysis-driven artificial molecular pump. Nature 594, 529-534 (2021).

${ }^{14}$ Wilson, M. R. et al. An autonomous chemically fuelled small-molecule motor. Nature 534, 235-240 (2016).

15 Astumian, R. D. How molecular motors work - insights from the molecular machinist's toolbox: the nobel prize in chemistry 2016. Chem. Sci. 8, 840-845 (2017).

16 Astumian, R. D. Kinetic asymmetry allows macromolecular catalysts to drive an information ratchet. Nat. Commun. 10, 3837 (2019).

17 Ragazzon, G. \& Prins, L. J. Energy consumption in chemical fuel-driven self-assembly. Nat. Nanotechnol. 13, 882-889 (2018).

18 Esposito, M. Open questions on nonequilibrium thermodynamics of chemical reaction networks. Commun. Chem. 3, $107(2020)$

19 Kelly, T. R., De Silva, H. \& Silva, R. A. Unidirectional rotary motion in a molecular system. Nature 401, 150-152 (1999).

20 Mock, W. L. \& Ochwat, K. J. Theory and example of a small-molecule motor. J. Phys. Org. Chem. 16, 175-182 (2003).

21 Fletcher, S. P., Dumur, F., Pollard, M. M. \& Feringa, B. L. A reversible, unidirectional molecular rotary motor driven by chemical energy. Science 310, 80-82 (2005).

${ }^{22}$ Collins, B. S. L., Kistemaker, J. C. M., Otten, E. \& Feringa, B. L. A chemically powered unidirectional rotary molecular motor based on a palladium redox cycle. Nat. Chem. 8, 860-866 (2016).

${ }^{23}$ Zhang, Y. et al. A chemically driven rotary molecular motor based on reversible lactone formation with perfect unidirectionality. Chem 6, 2420-2429 (2020).

${ }^{24}$ Borsley, S., Leigh, D. A. \& Roberts, B. M. W. A doubly kinetically-gated information ratchet autonomously driven by carbodiimide hydration. J. Am. Chem. Soc. 143, 4414-4420 (2021).

${ }^{25}$ Seifert, U. Stochastic thermodynamics, fluctuation theorems and molecular machines. Rep. Prog. Phys. 75, 126001 (2012).

${ }^{26}$ Parrondo, J. M. R. \& de Cisneros, B. J. Energetics of brownian motors: a review. Appl. Phys. A 75, 179-191 (2002).

27 Esposito, M., Lindenberg, K. \& Van den Broeck, C. Universality of efficiency at maximum power. Phys. Rev. Lett. 102, 130602 (2009).

${ }^{28}$ Benenti, G., Casati, G., Saito, K. \& Whitney, R. S. Fundamental aspects of steady-state conversion of heat to work at the nanoscale. Phys. Rep. 694, 1-124 (2017).

29 Jarzynski, C. Equalities and inequalities: Irreversibility and the second law of thermodynamics at the nanoscale. Annu. Rev. Condens. Matter Phys. 2, 329-351 (2011).

30 Peliti, L. \& Pigolotti, S. Stochastic Thermodynamics: An Introduction (Princeton University Press, Princeton, 2021).

31 Andrieux, D. \& Gaspard, P. Fluctuation theorems and the nonequilibrium thermodynamics of molecular motors. Phys. Rev. E 74, 011906 (2006).

32 Lipowsky, R. \& Liepelt, S. Chemomechanical coupling of molecular motors: Thermodynamics, network representations, and balance conditions. J. Stat. Phys. 130, 39-67 (2008).

33 Toyabe, S. et al. Nonequilibrium energetics of a single $\mathbf{f}_{1}$-atpase molecule. Phys. Rev. Lett. 104, 198103 (2010).

34 Ariga, T., Tomishige, M. \& Mizuno, D. Nonequilibrium energetics of molecular motor kinesin. Phys. Rev. Lett. 121, 218101 (2018).

35 Brown, A. I. \& Sivak, D. A. Theory of nonequilibrium free energy transduction by molecular machines. Chem. Rev. 120, 434-459 (2020).

${ }^{36}$ Ciliberto, S. Experiments in stochastic thermodynamics: Short history and perspectives. Phys. Rev. X 7, 021051 (2017).

37 Cover, T. M. \& Thomas, J. A. Elements of Information Theory (John Wiley \& Sons, Inc., Hoboken, 2012).

38 Parrondo, J. M. R., Horowitz, J. M. \& Sagawa, T. Thermodynamics of information. Nat. Phys. 11, 131-139 (2015).

${ }^{39}$ Horowitz, J. M. \& Esposito, M. Thermodynamics with continuous information flow. Phys. Rev. X 4, 031015 (2014).

${ }^{40}$ Leff, H. S. \& Rex, A. F. (eds.) Maxwell's Demon: Entropy, Information, Computing (Princeton University Press, Princeton, 1990).

41 Blackmond, D. G. "if pigs could fly" chemistry: A tutorial on the principle of microscopic reversibility. Angew. Chem. Int. Ed. 48, 2648-2654 (2009).

${ }^{42}$ Rao, R. \& Esposito, M. Nonequilibrium thermodynamics of chemical reaction networks: Wisdom from stochastic thermodynamics. Phys. Rev. X 6, 041064 (2016).

43 Penocchio, E., Rao, R. \& Esposito, M. Thermodynamic efficiency in dissipative chemistry. Nat. Commun. 10, 3865 (2019).

44 Wagoner, J. A. \& Dill, K. A. Mechanisms for achieving high speed and efficiency in biomolecular machines. Proc. Natl. Acad. Sci. U.S.A. 116, 5902-5907 (2019).

45 Howard, J. Protein power strokes. Curr. Biol. 16, R517 (2006).

${ }^{46}$ Hwang, W. \& Karplus, M. Structural basis for power stroke vs. brownian ratchet mechanisms of motor proteins. Proc. Natl. Acad. Sci. U.S.A. 116, 19777-19785 (2019). 
47 Astumian, R. D. Irrelevance of the power stroke for the directionality, stopping force, and optimal efficiency of chemically driven molecular machines. Biophys. J. 108, 291-303 (2015).

48 Pezzato, C., Cheng, C., Stoddart, J. F. \& Astumian, R. D. Mastering the non-equilibrium assembly and operation of molecular machines. Chem. Soc. Rev. 46, 5491-5507 (2017).

49 Das, K., Gabrielli, L. \& Prins, L. J. Chemically-fueled self-assembly in biology and chemistry. Angew. Chem. Int. Ed. 10.1002/anie.202100274 (2021).

50 Albaugh, A. \& Gingrich, T. R. Simulating a chemically-fueled molecular motor with nonequilibrium molecular dynamics. Preprint at http://arxiv.org/abs/2102.06298 (2021).

${ }^{51}$ Horowitz, J. M., Sagawa, T. \& Parrondo, J. M. R. Imitating chemical motors with optimal information motors. Phys. Rev. Lett. 111, 010602 (2013).

52 Fyfe, M. C. T. et al. Anion-assisted self-assembly. Angew. Chem. Int. Ed. Engl. 36, 2068-2070 (1997).

53 Astumian, R. D. Design principles for brownian molecular machines: how to swim in molasses and walk in a hurricane. Phys. Chem. Chem. Phys. 9, 5067-5083 (2007).

${ }^{54}$ Hartich, D., Barato, A. C. \& Seifert, U. Stochastic thermodynamics of bipartite systems: transfer entropy inequalities and a maxwell's demon interpretation. J. Stat. Mech.: Theory Exp. 2014, P02016 (2014).

${ }^{55}$ Kondepudi, D. K. \& Prigogine, I. Modern Thermodynamics: From Heat Engines to Dissipative Structures (John Wiley \& Sons, Ltd., Chichester, 2015).

56 Astumian, R. \& Bier, M. Mechanochemical coupling of the motion of molecular motors to atp hydrolysis. Biophysical Journal 70, 637-653 (1996).

57 Hill, T. L. Free energy transduction in biology (Academic Press, New York, 1977).

58 Alvarez-Pérez, M., Goldup, S. M., Leigh, D. A. \& Slawin, A. M. Z. A chemically-driven molecular information ratchet. J. Am. Chem. Soc. 130, 1836-1838 (2008).

${ }^{59}$ Carlone, A., Goldup, S. M., Lebrasseur, N., Leigh, D. A. \& Wilson, A. A three-compartment chemically-driven molecular information ratchet. J. Am. Chem. Soc. 134, 8321-8323 (2012).

${ }^{60}$ Lussis, P. et al. A single synthetic small molecule that generates force against a load. Nat. Nanotechnol. 6, 553-557 (2011).

61 Amano, S., Borsley, S., Leigh, D. A. \& Sun, Z. Chemical engines: Driving systems away from equilibrium through catalyst reaction cycles. Submitted for publication (2021).

${ }^{62}$ Kay, E. R., Leigh, D. A. \& Zerbetto, F. Synthetic molecular motors and mechanical machines. Angew. Chem. Int. Ed. 46, 72-191 (2007).

${ }^{63}$ Katz, S., Lebowitz, J. L. \& Spohn, H. Phase transitions in stationary nonequilibrium states of model lattice systems. Phys. Rev. B 28, 1655-1658 (1983).

64 Esposito, M. Stochastic thermodynamics under coarse graining. Phys. Rev. E 85, 041125 (2012).

65 Maes, C. Local detailed balance. Preprint at https://arxiv.org/abs/2011.09200 (2021). 